Citation: Çelik, H . "Applications of Weighted Arithmetic-Geometric Means Inequality to Functional Inequalities". Journal of Engineering Technology and Applied Sciences 2 (1) 2017 : 27-31

\title{
APPLICATIONS OF WEIGHTED ARITHMETIC GEOMETRIC MEANS INEQUALITY TO FUNCTIONAL INEQUALITIES
}

\author{
Halil İbrahim Çelik \\ Department of Mathematics, Faculty of Arts and Sciences, Marmara University, Istanbul, Turkey \\ hicelik@marmara.edu.tr
}

\begin{abstract}
In this paper, a functional inequality is proven and the result is illustrated with some elementary functions.
\end{abstract}

Keywords: Arithmetic-geometric means inequality, Young inequality, extremum values, functional inequalities, elementary functions, monotone sequences.

\section{Introduction}

Let $f$ be a positive real function defined on a subset $E$ of the real line $\mathbb{R}$.The function $h: E \rightarrow$ $\mathbb{R}, h(x)=f(x)^{f(x)}$ is defined by $h(x)=e^{f(x) \ln f(x)}$ where the function $\ln x$ is the natural logarithm function. If $f$ and $g$ are two such functions defined on the set $E$, then it is a natural problem to compare the values $f(x)^{f(x)}$ and $g(x)^{g(x)}$ for each $x \in E$. Since the arithmetic and geometric means inequality is a very important elementary tool for making estimates or approximation it is expected that the inequality play a role for the solution of these types of problems.

Let $W=\left\{\lambda=\left(\lambda_{1}, \lambda_{2}, \ldots, \lambda_{n}\right) \in \mathbb{R}^{n}: \lambda_{j} \in[0,1]\right.$ for $\left.j=1,2, \ldots, n, \sum_{j=1}^{n} \lambda_{j}=1\right\}$ be the set of weight vectors and $P=\left\{x=\left(x_{1}, x_{2}, \cdots x_{n}\right) \in \mathbb{R}^{n}\right.$ : for $\left.j=1,2, \cdots, n, x_{j}>0\right\}$ be the set of positive vectors.

Definition 1.1. Let $\lambda \in W$ and $x \in P$. The real number

$$
A_{n}(x, \lambda)=\lambda_{1} x_{1}+\lambda_{2} x_{2}+\cdots+\lambda_{n} x_{n}
$$

is called the weighted arithmetic mean of $n$ positive real numbers $x_{1}, x_{2}, \cdots, x_{n}$ of weight $\lambda$ and the number 
$G_{n}(x, \lambda)=x_{1}^{\lambda_{1}} \cdot x_{2}^{\lambda_{2}} \cdots x_{n}^{\lambda_{n}}$

is called the weighted geometric mean of $n$ positive real numbers $x_{1}, x_{2}, \cdots, x_{n}$ of weight $\lambda$.

For the weight vector $\lambda=\left(\frac{1}{n}, \frac{1}{n}, \cdots, \frac{1}{n}\right)$

$A_{n}(x, \lambda)=A_{n}=\frac{1}{n}\left(x_{1}+x_{2}+\cdots+x_{n}\right)$

is the arithmetic mean of the $n$ positive real numbers $x_{1}, x_{2}, \cdots, x_{n}$ and

$$
G_{n}(x, \lambda)=G_{n}=\left(x_{1} \cdot x_{2} \cdots x_{n}\right)^{\frac{1}{n}}=\sqrt[n]{x_{1} \cdot x_{2} \cdots x_{n}}
$$

is the geometric mean of the $n$ positive real numbers $x_{1}, x_{2}, \cdots, x_{n}$.

We have the following inequality between arithmetic and geometric means.

Theorem 1.2 (Arithmetic and geometric means inequality $[2,5]) . G_{n}(x, \lambda) \leq A_{n}(x, \lambda)$ for all positive real numbers $x_{1}, x_{2}, \cdots, x_{n}$ and for all $\lambda \in W$ and the inequality is strict unless $x_{1}=$ $x_{2}=\cdots=x_{n}$.

Arithmetic-geometric means inequalities has numerous applications in mathematics and other areas. We note the following example which shows that certain type of extremum value problems can be resolved easily by using this inequality.

Example 1.3. We will find the minimum value of the function

$$
f(x, y, z)=\frac{\sin x}{\sin y}+\sqrt{\frac{\sin y}{\sin z}}+\sqrt[3]{\frac{\sin z}{\sin x}} \text { over the set } \Omega=\left\{(x, y, z) \in \mathbb{R}^{3}: x, y, z \in \mathbb{R}\right\} .
$$

Solution. From the arithmetic and geometric means inequality for every $(x, y, z) \in \Omega$ we have

$$
\begin{gathered}
f(x, y, z)=\frac{\sin x}{\sin y}+\sqrt{\frac{\sin y}{\sin z}}+\sqrt[3]{\frac{\sin z}{\sin x}} \\
=\frac{\sin x}{\sin y}+\frac{1}{2} \sqrt{\frac{\sin y}{\sin z}}+\frac{1}{2} \sqrt{\frac{\sin y}{\sin z}}+\frac{1}{3} \sqrt[3]{\frac{\sin z}{\sin x}}+\frac{1}{3} \sqrt[3]{\frac{\sin z}{\sin x}}+\frac{1}{3} \sqrt[3]{\frac{\sin z}{\sin x}} \geq 6 \sqrt[6]{\frac{1}{4} \frac{1}{27} \frac{\sin x}{\sin y} \frac{\sin y \sin z}{\sin z} \frac{\sin z}{1 / 3}} \\
=2^{2 / 3} 3^{1 / 2}
\end{gathered}
$$

Since the equality occurs when $\frac{\sin x}{\sin y}=\frac{1}{2} \sqrt{\frac{\sin y}{\sin z}}=\frac{1}{3} \sqrt[3]{\frac{\sin z}{\sin x}}$ or equivalently when $\sin y=$ $\sqrt{3} \sqrt[3]{2}$ and $\sin z=\frac{3}{2} \sqrt{3} \sin x$ by the definition of minimum value it follows that $\min \{f(x, y, z):(x, y, z) \in \Omega\}=2^{2 / 3} 3^{1 / 2}$.

For positive real numbers $a$ and $b$ and for $\lambda \in(0,1)$ the weighted arithmetic-geometric means inequality is the inequality 


$$
a^{\lambda} b^{1-\lambda} \leq \lambda a+(1-\lambda) b
$$

For $p>1$ if we take $\lambda=\frac{1}{p}, 1-\lambda=\frac{1}{q}$ and replace $a$ with $a^{p}$ and $b$ with $b^{q}$ we get the Young's inequality $a b \leq \frac{a^{p}}{p}+\frac{b^{q}}{q}$.

This inequality is one of the most important inequality in mathematics because the famous Cauchy, Hölder and Minkowski inequalities follow from it.

\section{The functional inequality and applications}

Theorem 2.1. Let $f, g$ be positive real valued functions defined on a subset Eof the real line $\mathbb{R}$. Then $f(x)^{f(x)} \leq g(x)^{g(x)}$ if $f(x) \leq g(x)$ and $f(x)+g(x) \geq 1$ for each $x \in E$. The inequality is strict if $f(x)<g(x)$ and $f(x)+g(x) \geq 1$ and the equality occurs if and only if $\frac{f(x)}{g(x)}=\frac{\ln (g(x))}{\ln (f(x))}$ for $x \in E$.

Proof. Let $x \in E$ be arbitrary, $a=f(x), b=f(x)+g(x)$ and $\lambda=\frac{f(x)}{g(x)}$. Then $a>0, b>0$ and $\lambda \in(0,1]$. Since $1-\lambda \geq 0$ and $f(x)+g(x) \geq 1$ by the weighted arithmetic and geometric means inequality

$$
\begin{gathered}
f(x)^{\frac{f(x)}{g(x)}} \leq f(x)^{\frac{f(x)}{g(x)}}(f(x)+g(x))^{1-\frac{f(x)}{g(x)}} \\
\leq \frac{f(x)}{g(x)} f(x)+\left(1-\frac{f(x)}{g(x)}\right)(f(x)+g(x))=g(x) .
\end{gathered}
$$

It follows that $f(x)^{f(x)} \leq g(x)^{f(x)}$ and the inequality is strict when $f(x)<g(x)$ and $f(x)+$ $g(x) \geq 1$.

Remark 2.2. 1) In the proof of the Theorem 2.1 we cannot directly use the properties of the convex function $\varphi:(0, \infty) \rightarrow \mathbb{R}, \varphi(x)=x^{x}=e^{x \ln x}$ since this function has the global minimum value at the point $x=\frac{1}{e}$. But since the function $\varphi(x)=x^{x}$ is monotone increasing on $\left[\frac{1}{e}, \infty\right)$ the inequality is trivial when $\frac{1}{e} \leq f(x) \leq g(x)$.

2) Theorem 2.1 holds true for positive real valued function defined on arbitrary sets not just on the subsets of the real line.

By mathematical induction we get the following result.

Corollary 2.3. For $j=1,2, \cdots, n$ let $f_{j}, g_{j}$ be positive real valued functions defined on a subset Eof the real line $\mathbb{R}$. Then $\prod_{j=1}^{n} f_{j}(x)^{f_{j}(x)} \leq \prod_{j=1}^{n} g_{j}(x)^{g_{j}(x)}$ if $f_{j}(x) \leq g_{j}(x)$ and $f_{j}(x)+$ $g_{j}(x) \geq 1$ for each $x \in E$ and $j=1,2, \cdots, n$ The inequality is strict if $f_{j}(x)<g_{j}(x)$ and $f_{j}(x)+g_{j}(x) \geq 1$ and the equality occurs if and only if $\frac{f_{j}(x)}{g_{j}(x)}=\frac{\ln g_{j}(x)}{\ln f_{j}(x)}$ for $x \in$ Eand $j=$ $1,2, \cdots, n$.

For the polynomial functions we have the following result. 
Corollary 2.4. Let $p$ and $q$ be real polynomial functions such that $m=$ degp $\leq$ degq $=n$. If $0 \leq p(0) \leq q(0), 0 \leq p^{(j)}(0) \leq q^{(j)}(0)$ for $j=1,2, \cdots, n$ and $p(0)+q(0) \geq 1$, where $p^{(j)}(0)$ and are $q^{(j)}(0)$ the $j^{\text {th }}$ derivatives of $p$ and $q$ at $x=0$ respectively, then $p(x)^{p(x)} \leq$ $q(x)^{q(x)}$ for all $x \in[0, \infty)$.

Proof. From the hypothesis we have $p(x) \leq q(x)$ and $p(x)+q(x) \geq p(0)+q(0) \geq 1$ for all $x \in[0, \infty)$. So the inequality follows from the Theorem 2.1 .

The following result is the problem 32 given in [4].

Proposition 2.5. For all $x \in\left(0, \frac{\pi}{4}\right)(\sin x)^{\sin x}<(\cos x)^{\cos x}$.

Proof. Since $\sin x<\cos x$ and $\sin x+\cos x=\sqrt{2} \cos \left(\frac{\pi}{4}-x\right)>\sqrt{2} \cos \frac{\pi}{4}=1$ for all $x \in$ $\left(0, \frac{\pi}{4}\right)$ the inequality follows from the Theorem 2.1 .

A similar reasoning gives the inequality $(\cos x)^{\cos x}<(\sin x)^{\sin x}$ for all real numbers $x \in$ $\left(\frac{\pi}{4}, \frac{\pi}{2}\right)$. Since the functions $\cos x$ and $\sin x$ are $2 \pi$-periodic functions these inequalities hold true on $2 \mathrm{n} \pi$ translations of $\left(0, \frac{\pi}{4}\right)$ and $\left(\frac{\pi}{4}, \frac{\pi}{2}\right)$ for each $n \in \mathbb{Z}$.

Proposition 2.6. For all $x \in\left(0, \frac{\pi}{4}\right)(\tan x)^{\tan x}<(\cot x)^{\cot x}$

Proof. Since $\tan x+\cot x=\frac{2}{\sin 2 x} \geq 2>1$ and $\tan x<\cot x$ for all $x \in\left(0, \frac{\pi}{4}\right)$ the inequality follows from the Theorem 2.1.

A similar argument gives the inequality $(\cot x)^{\cot x}<(\tan x)^{\tan x}$ for all $x \in\left(\frac{\pi}{4}, \frac{\pi}{2}\right)$ and since the functions $\tan x$ and $\cot x$ are $\pi$-periodic functions these inequalities are true for $n \pi$ translations of $\left(0, \frac{\pi}{4}\right)$ and $\left(\frac{\pi}{4}, \frac{\pi}{2}\right)$ for each $n \in \mathbb{Z}$.

Proposition 2.7. For all $x \in(0, \infty)(\sinh x)^{\sinh x}<(\cosh x)^{\cosh x}$.

Proof. Since

$\sinh x=\frac{1}{2}\left(e^{x}-e^{-x}\right)<\frac{1}{2}\left(e^{x}+e^{-x}\right)=\cosh x$ and $\sinh x+\cosh x=e^{x}>1$ for all $x \in$ $(0, \infty)$ the inequality follows from the Theorem 2.1 .

For the inverse hyperbolic functions $\cosh ^{-1} x=\ln \left(x+\sqrt{x^{2}-1}\right)$ and $\sinh ^{-1} x=\ln (x+$ $\left.\sqrt{x^{2}+1}\right)$ we have the following result: By the first derivative test the function $\varphi(x)=$ $\left(x+\sqrt{x^{2}+1}\right)\left(x+\sqrt{x^{2}-1}\right)$ is strictly monotone increasing on $[1, \infty)$. Since $\varphi(1)=1+$ $\sqrt{2}<e$ by the intermediate value theorem for a continuous function there is a unique point $c_{e} \in$ $(1, \infty)$ such that $\varphi\left(c_{e}\right)=e$.

Proposition 2.8. For all $x \in\left[c_{e}, \infty\right)\left(\cosh ^{-1} x\right)^{\cosh ^{-1} x}<\left(\sinh ^{-1} x\right)^{\sinh ^{-1} x}$.

Proof. Since $\cosh ^{-1} x=\ln \left(x+\sqrt{x^{2}-1}\right)<\ln \left(x+\sqrt{x^{2}+1}\right)=\sinh ^{-1} x$ and 


$$
\begin{aligned}
& \sinh ^{-1} x+\cosh ^{-1} x=\ln \left(x+\sqrt{x^{2}+1}\right)+\ln \left(x+\sqrt{x^{2}-1}\right) \\
& =\ln \left(x+\sqrt{x^{2}+1}\right)\left(x+\sqrt{x^{2}-1}\right) \geq \ln \left(c_{e}+\sqrt{c_{e}^{2}+1}\right)\left(c_{e}+\sqrt{c_{e}^{2}-1}\right) \\
& =\ln e=1
\end{aligned}
$$

for all $x \in\left[c_{e}, \infty\right)$ the inequality follows from the Theorem 2.1 .

Theorem 2.1 can also be applied to show that certain sequences of real numbers are monotone increasing.

Example 2.9. The sequence $\left(\left(1-\frac{1}{n-1}\right)^{\left(1-\frac{1}{n-1}\right)}\right)_{n=3}^{\infty}$ is monotone increasing.

Solution. Let $3 \leq n \in \mathbb{N}$ be arbitrary. Since $1-\frac{1}{n-1}<1-\frac{1}{n}$ and $1-\frac{1}{n-1}+1-\frac{1}{n}=2-\frac{2 n-1}{n(n-1)} \geq 2-\frac{2 n-1}{2 n}>1$ by Theorem 2.1 we have

$$
\left(1-\frac{1}{n-1}\right)^{\left(1-\frac{1}{n-1}\right)}<\left(1-\frac{1}{n}\right)^{\left(1-\frac{1}{n}\right)}
$$

Therefore the sequence $\left(\left(1-\frac{1}{n-1}\right)^{\left(1-\frac{1}{n-1}\right)}\right)_{n=3}^{\infty}$ is monotone increasing.

We end the paper with the following question.

Question. Can the hypothesis $f(x)+g(x) \geq 1$ weakened and is there a best constant smaller than 1 for the functional inequality $f(x)^{f(x)}<g(x)^{g(x)}$ to hold?

\section{References}

[1] G. H. Hardy, J. E. Littlewood and G. Polya, Inequalities, 2nd. ed., Cambridge, New York 1959.

[2] N. D. Kazarinoff, Analytic Inequalities, Holt, Rinehart and Winston, New York, 1961.

[3] I. J. Maddox, Elements of Functional Analysis, Second ed., Cambridge Univ. Press, 1988.

[4] Thomas.J. Mildford, Olympiad Inequalites, 2006, http://www.unl.edu/amc

[5] W. Rudin, Real and Complex Analysis, McGraw-Hill Book Company, New York, 1987. 